\title{
Improving mathematical models of cancer by including resistance to therapy: a study in non-small cell lung cancer
}

\author{
Virginia Ardévol Martinez ${ }^{a}$, Narmin Ghaffari Laleh $^{b}$, \\ Monica Salvioli ${ }^{a, c}$, Frank Thuijsman ${ }^{a}$, Joel S. Brown ${ }^{d}$, \\ Rachel Cavill ${ }^{a}$, Jakob Nikolas Kather $^{b}$ and Kateřina Staňkovác \\ ${ }^{a}$ Maastricht University, Maastricht, The Netherlands \\ ${ }^{b}$ University Hospital RWTH Aachen, Aachen, Germany \\ ${ }^{c}$ Delft University of Technology, Delft, The Netherlands \\ ${ }^{d}$ Moffitt Cancer Center, Tampa, Florida, United States of America
}

\begin{abstract}
In this paper, a large dataset of 590 Non-Small Cell Lung Patients treated with either chemotherapy or immunotherapy was used to determine whether a game-theoretic model including both evolution of therapy resistance and cost of resistance provides a better fit than classical mathematical models of population growth (exponential, logistic, classic Bertalanffy, general Bertalanffy, Gompertz, general Gompertz). This is the first time a large clinical patient cohort (as opposed to only in-vitro data) has been used to apply a game-theoretic cancer model. The game-theoretic model provides a better fit to the tumor dynamics of the 590 Non-Small Cell Lung Cancer patients than any of the non-evolutionary population growth models. This is not simply due to having more parameters in the game-theoretic model. The game-theoretic model is able to fit accurately patients whose tumor burden exhibit a U-shaped trajectory over time. We then demonstrate how this game-theoretic model provides predictions of tumor growth based on just a few initial measurements. Assuming that treatment-specific parameters define the treatment impact completely, we then explore alternative treatment protocols and their impact on the tumor growth. As such, the model can be used to suggest patient-specific optimal treatment regimens with the goal of minimizing final tumor burden. Therapeutic protocols based on game-theoretic modeling can predict tumor growth, and improve patient outcome. The model invites evolutionary therapies that anticipate and steer the evolution of therapy resistance.
\end{abstract}

Keywords: Evolutionary game theory, Non-Small Cell Lung Cancer, immunotherapy, radiotherapy, Stackelberg evolutionary game, resistance

\section{Introduction}

Lung cancer is the second most common cancer and the leading cause of cancer death for both men and women [4]. Non-Small Cell Lung Cancer (NSCLC) is the most frequent type of lung cancer, accounting for $84 \%$ of all lung cancer diagnoses. Although novel anti-cancer 
therapies like targeted therapies and immunotherapy are allowing people with metastatic lung cancer to live longer than ever before, metastatic lung cancer remains incurable [4, 3]. This is often caused by therapy resistance [11, 20, 16, 13].

In order to to improve therapy, we need to understand tumor response to different possible therapy options. Mathematical modelling helps with this understanding [1]. Here we compare the fits of six mathematical models (exponential, logistic, classic Bertalanffy, general Bertalanffy, Gompertz and general Gompertz) to quantitative measurements of tumor diameter changes over time from patients with Non-Small Cell Lung Cancer with a novel game-theoretic model. Recently, Ghaffari Laleh et al (2021) have fitted classic models of tumor growth to this dataset and performed a comparison between them, showing that they can fit the trajectory of tumor growth relatively well. They provide a good estimation of the future response based on early treatment data. However, their predictive capabilities fail when tumor growth is not monotonic, i.e. for tumors that are neither continuously growing nor shrinking [12]. Yet, many tumor growth dynamics show a U-shape where therapy is initially effective but loses efficacy as the cancer cell population evolves resistance.

In this paper, we use a subset of 590 patients from this data set, corresponding to patients with NSCLC treated with chemotherapy or immunotherapy for which we have measurements for at least 6 time points. We propose a game-theoretic model which assumes that evolution of therapy resistance is a quantitative trait. This is different from some existing models that assume a qualitative trait where different cancer cell types are either resistant or sensitive to treatment [22, 14, 3]. The model we utilize here has been proposed in [16] and further studied in [19]. We will verify whether this mode outperforms the classical models of tumor growth when fitted with the NSCLC patient data t [12].

After fitting the model to the data, we analyze whether different treatment protocols would have been better for some of the patients, This application illustrates an application of Stackelberg evolutionary game theory. Besides improving the accuracy for predicting tumor dynamics and the evolution of resistance under treatment, the model provides a successful application of evolutionary game theory to describe and optimize cancer treatment.

This paper is organized as follows. In Section 2, we introduce the model and fit it with a subset of the anonymized data used in [12. The subset of patients with metastatic NSCLC were treated with either immune checkpoint inhibition or chemotherapy. In Section 3 , we evaluate the results obtained, as well as the accuracy of the model in predicting the future dynamics of the tumor. We compare our results with those given by the classical population growth models explored in [12. Moreover, we show how our model can be used to design alternative therapies for optimizing any pre-specified treatment goals. We can than compare the possible patient outcomes had the patients undergone a different treatment, as well as to explore other theoretical scenarios, such as the effect of treatments targeting the evolution of resistance. Section 4 concludes by summarizing the main outcomes and discussing limitations and future directions.

\section{Material and methods}

\subsection{Game-theoretic model}

Here we model the cancer cells' response to treatment as an evolutionary game as in [16]. The physician has one decision variable $m$, which represents either the dose of Docetaxel, a 
chemotherapy drug, or an immune checkpoint inhibitor that targets PD-1 (MPDL3280A). As no patients in our data set received both of the treatments together, we use the same model for both therapeutic options, with a slightly different assumption on the treatment dose $m$. In the case of chemotherapy, the physician can regulate the drug dose and $m$ exists on a continuum between 0 and 1, where the standard of care corresponds to $m=1$ and $m=0$ means that no treatment is given. On the other hand, in the case of immunotherapy, the physician only has two options: to apply the treatment $(m=1)$ or not $(m=0)$. This is because a higher dose of immunotherapy does not necessarily have a proportional effect on the cancer population, as investigated in [15]. For both treatments, when fitting the model to the patient data, we assume that the dose is fixed and $m=1$ when the treatment is applied and $m=0$ otherwise. However, when looking for alternative treatments, we may vary $m$ between 0 and 1 in the case of chemotherapy. We model the cancer's eco-evolutionary dynamics by means of a population vector $x$ of cancer cells, with therapy resistance as a continuous trait $u \in[0,1]$, where $u$ indicates the intensity of therapy resistance: cells with $u=0$ are completely susceptible (sensitive) to the treatment, while cells with $u=1$ are fully resistant to the treatment [17, 19, 16].

In order to integrate the time scales of the ecological and the evolutionary dynamics we use a general approach, called Darwinian dynamics, where the population dynamics and the dynamics of the evolving trait are modeled using a fitness-generating function [5, 21]. We assume that the strategies of the individual cells in the evolving population are inherited and their payoffs depend on the expected fitness (representing per capita growth rates), which we denote as $G(u, x, m)$ and refer to as the fitness-generating function. The evolutionary dynamics are defined as

$$
\dot{x}=x G(u, x, m) .
$$

The fitness function also determine the evolutionary dynamics that describe how the cancer cells level of resistance evolves in response to the physician's treatment choice and are defined as

$$
\dot{u}=\sigma \frac{\partial G}{\partial u}
$$

where $\sigma$ determines evolutionary speed, which can be seen as increasing with additive genetic variance or the mutation rate. The G-function approach allows us to determine how natural selection will act on a population's ecological and evolutionary dynamics.

While the function $G(u, x, m)$ could have different forms, here we confine ourselves to the form used in the existing literature [17, 19, 16]:

$$
G(u, x, m)=r\left(1-\frac{x}{K(u)}\right)-\frac{m}{k+b u}-d .
$$

The first term of (3), $r\left(1-\frac{x}{K(u)}\right)$, refers to the per capita growth rate of the cancer cells, where $r$ represents the intrinsic growth rate, $x$ the population of cancer cells, $u$ their resistance strategy and $K(u)$ the carrying capacity, which is assumed to be a function of the resistance trait . In particular, similarly to [23, 6], we assume that there is a cost of resistance manifested in the carrying capacity such that $K(u)$ decreases as $u$ increases: $K(u)=K_{\max } e^{-g u}$, where $K_{\max }$ is a constant that indicates the maximum possible carrying capacity and $g$ determines the magnitude of the cost of resistance. This is due to the fact that, in general, resistance appears in the form of cells that are not sensitive to the 
treatment, and the maintenance of these resistance mechanisms might require extra energy that cannot be devoted to proliferation, making resistant cells less efficient at utilizing resources for survival and proliferation in the absence of treatment [10]. The second term of the G-function, $\frac{m}{k+b u}$, represents the mortality due to the treatment, where $m$ is the treatment dose, $k$ is innate resistance, and $b$ is the benefit of the resistance trait in reducing drug efficicacy. The background rate of cell death is given by $d$. Table 1 summarizes all parameters, their definitions and values.

\begin{tabular}{clll}
\hline Variables & Meaning & Values & \\
\hline$x$ & Cancer cell population & in interval $[0,1]$ & \\
$u$ & Resistance strategy & non-negative & \\
$m$ & Treatment dose & in interval $[0,1]$ & \\
\hline Parameters & Meaning & Values & Variability \\
\hline$d$ & Intrinsic death rate & in interval $[0,1)$ & treatment-specific \\
$r$ & Growth rate of the cancer cells & in interval $[d, 1)$ & patient-specific \\
$K_{\max }$ & Maximum carrying capacity & nonnegative & treatment-specific \\
$k$ & Innate cell immunity & in interval $[0,20]$ & treatment-specific \\
$b$ & Magnitude of resistance benefit & in interval [0,20] & treatment-specific \\
$\sigma$ & Evolutionary speed & in interval $[0,0.1]$ & patient-specific \\
$g$ & Magnitude of cost of resistance & in interval $[0,1)$ & treatment-specific \\
\hline
\end{tabular}

Table 1: Variables and parameters of the model.

\subsection{Data pre-processing}

We used data sets from four different clinical trials, namely the four trials from [12] which correspond to NSCLC. All the data correspond to patients with metastatic NSCLC treated with Atezolizumab (previously known as MPDL3280A), which is an immune checkpoint inhibitor directed against the Programmed Death Ligand 1 (PD-L1), or with Docetaxel, a chemotherapeutic agent. The data used consists of the one-dimensional longest diameter of target and non-target lesions manually measured on CT scans. We cleaned and preprocessed the data following the guidelines in [12] in order to be able to compare the final results.

We selected the primary tumor (target lesion) of each patient and considered only those tumors for which more than 6 observations are available, to enable a more robust fitting of the mathematical model to the data and avoid overfitting. The cohort of patients with valid data consisted of 590 patients. Patient varied in the number of time for the selected target lesion. The distribution of patients among studies and treatment arms is explained in more details in [12].

In order to estimate the population of cancer cells of each tumor from the measurements of the longest diameter $(L D)$, the tumor volume $(V)$ was calculated using the formula $V=0.5 \cdot(L D)^{3}$, following common practice in tumor modelling when only a single tumor measurement is available [9].

Furthermore, as the range of tumor sizes wasvery large, going from 0 to $5926176 \mathrm{~mm}^{3}$, the 
cell populations were rescaled in the range 0 to 1 , using the formula

$$
x=\frac{x_{\mathrm{old}}-x_{\min }}{x_{\max }-x_{\min }}
$$

where $x$ represents the rescaled value, $x_{\text {old }}$ is the original value of the cell population (estimated from the diameter), and $x_{\min }$ and $x_{\max }$ represent the minimum and maximum values of the cell populations among the whole dataset, respectively. This was done in order to have only three patient-specific parameters in the model and therefore all other parameters are fixed for all of the patients.

We then categorized the tumors into four treatment response groups/categories: "Up", "Down", "Fluctuate" and "Evolution". For this purpose, for each tumor we created a vector containing the differences between each $L D$ measurement at time point $t_{k+1}$ and the previous measurement at time point $t_{k}$, for all the time points where a measurement was taken. If the $L D$ at $t_{k+1}$ is bigger than at $t_{k}$, we consider the difference as positive and vice versa. The tumors were classified and categorized as follows:

- The "U" category includes patients whose difference vector values are always positive and patients with a positive difference after the first measurement if the ratio between the sum of all positive values to the sum of all negative values is greater than 2 .

- The "Down" category includes patients whose difference vector values are always negative or a negative difference after the first measurement if the overall ratio between the sum of all negative values to the sum of all positive values is greater than 2 .

- The "Evolution" category includes patients who initially respond to treatment and then start experiencing tumor progression. More precisely, we include tumors for which the first two values of the difference vector are negative, the maximum of the volume corresponds to the first or the last time point, and the sum of the last two values of the difference vector is greater than -1 times the first value of the vector divided by 2 (i.e., the evolution of resistance causes the tumor to grow at least half as much as it shrinked at the start of the treatment).

- The "Fluctuate" category contains all patients who correspond to none of the previous categories.

The classes "Up" and "Down" correspond to those described in [12, while the class "Fluctuate" defined in [12] is now divided into two classes: "Evolution" and "Fluctuate". This was done because tumors in the class "Evolution" are more likely to exhibit a U-shape due to the evolution of treatment-induced resistance. Since our model takes evolution of therapy resistance explicitly into account, we want to confirm the hypothesis that it fits the tumors in this category more accurately than the other models.

\subsection{Fitting the model}

Given that the only information available is the value of $x$ at certain time points and whether the patient is subject to treatment at that time point or not, we need to estimate all the parameters introduced in Table 1 in order to fit the model.

Parameter estimation is done using GEKKO v0.2.8, a Python package for machine learning and optimization, specialized in dynamic optimization of differential algebraic equations [2]. 
As there are seven free parameters, estimating their values poses a significant challenge. We consider two of the parameters and the initial condition for $u$ to be patient-specific and fix the rest, as summarized in Table 1. Initial experimentation suggested that the best option is to estimate $r$ and $\sigma$ per patient and fix the remaining parameters.

Thus, to fit the model, the parameters $b, k, K_{\max }, g$, and $d$ are fitted with assumption that they are treatment-specific, meaning that they are fixed to a selected set of values for all the patients who received the same treatment, while $\sigma, r$ and the initial value of $u$ are are assumed to be patient-specific, that is, they are estimated separately for each tumor with the objective of minimizing the mean squared error (MSE) between the real data and the predictions given by the model. For all tests, we assume $m=1$ when treatment is applied. The values of the parameters which are not patient-specific are selected after performing a grid search to find the combination that gives the best results. The model is fitted to the data for each tumor for each combination of parameters and then, the mean squared error is evaluated per tumor. The final value of the average error of all the tumors is then used to select the best parameters.

\subsection{Separating patients into different clusters}

Due to the fact that the behaviours and volumes of the tumors vary significantly from patient to patient and the number of parameters to optimize is relatively large, we separate the tumors in different clusters such that each cluster has different parameter values.

First of all, as we mentioned before, the parameters $b, k, K_{\max }, g$, and $d$ are assumed to be treatment-specific, so different values are estimated for each of the two treatments. Within each treatment group, we further stratify the patients according to the following criteria.

The first clustering criterion is the initial volume of the tumors, as the range of the volumes is considerably large and the volume of the tumor might have an impact on its evolution and thus on the parameters. Moreover, this information can be extracted solely from the first data point, so it poses advantages for prediction.

The second clustering criterion is the initial trend of the growth of the tumors, that is, whether they increase or decrease once treatment starts. This is a reasonable criterion as the first reaction to treatment may contain valuable information about the evolution of the tumor and it can be measured with only the first two data points.

Therefore, for each treatment group, tumors are separated into four clusters according to initial volume - Size 1, Size 2, Size 3 and Size 4 - and two sub-clusters according to the initial trend - increasing and decreasing -, so in total we differentiate eight groups per treatment, corresponding to all the possible combinations of initial trend and initial volume. Tumors are classified as Size 1 if their volume is smaller than $1021.9 \mathrm{~mm}^{3}$, Size 2 if their volume is between $1021.9 \mathrm{~mm}^{3}$ and $10219 \mathrm{~mm}^{3}$, Size 3 if it is between $10219 \mathrm{~mm}^{3}$ and 61314.24 $\mathrm{mm}^{3}$ and Size 4 if the volume surpasses the latter threshold. On the other hand, tumors are included in the increasing group if the second measurement of the diameter is bigger or equal than the first one, and in the decreasing group if it is smaller.

For each group, a grid search is performed to fit the parameters, where for each grid point patient-specific fit is carried out. The parameters that lead to the best fit per group are selected. Their values are summarized in Table 2. 


\begin{tabular}{ccc}
\hline & Increasing & Decreasing \\
& $b=20$ & $b=2$ \\
Size 1 & $k=0.2$ & $k=0.2$ \\
$\left(V \leq 1021.9 \mathrm{~mm}^{3}\right)$ & $K_{\max }=1$ & $K_{\max }=1$ \\
& $g=0.9$ & $g=0.1$ \\
& $d=0.01$ & $d=0.2$ \\
\hline & $b=2$ & $b=1$ \\
Size 2 & $k=1$ & $k=0.9$ \\
$\left(1021.9 \mathrm{~mm}^{3}<V\right.$ & $K_{\max }=1$ & $K_{\max }=1$ \\
$\left.\leq 10219 \mathrm{~mm}^{3}\right)$ & $g=0.9$ & $g=0.1$ \\
& $d=0.1$ & $d=0.2$ \\
\hline & $b=2$ & $b=1$ \\
Size 3 & $k=10$ & $k=0.9$ \\
$\left(10219 \mathrm{~mm}^{3}<V\right.$ & $K_{\max }=1$ & $K_{\max }=1$ \\
$\left.\leq 61314.24 \mathrm{~mm}^{3}\right)$ & $g=0.9$ & $g=0.1$ \\
& $d=0.2$ & $d=0.1$ \\
\hline & $b=20$ & $b=20$ \\
Size 4 & $k=1$ & $k=0.9$ \\
$\left(V>61314.24 \mathrm{~mm}^{3}\right)$ & $K_{\max }=1$ & $K_{\max }=1$ \\
& $g=0.9$ & $g=0.9$ \\
& $d=0.01$ & $d=0.1$ \\
\hline
\end{tabular}

\begin{tabular}{ccc}
\hline & Increasing & Decreasing \\
& $b=1$ & $b=1$ \\
Size 1 & $k=2$ & $k=1$ \\
$\left(V \leq 1021.9 \mathrm{~mm}^{3}\right)$ & $K_{\max }=1$ & $K_{\max }=1$ \\
& $g=0.5$ & $g=0.1$ \\
& $d=0.01$ & $d=0.1$ \\
\hline & $b=0.2$ & $b=1$ \\
Size 2 & $k=2$ & $k=0.9$ \\
$\left(1021.9 \mathrm{~mm}^{3}<V\right.$ & $K_{\max }=1$ & $K_{\max }=1$ \\
$\left.\leq 10219 \mathrm{~mm}^{3}\right)$ & $g=0.9$ & $g=0.1$ \\
& $d=0.01$ & $d=0.2$ \\
\hline & $b=2$ & $b=20$ \\
Size 3 & $k=0.9$ & $k=0.2$ \\
$\left(10219 \mathrm{~mm}^{3}<V\right.$ & $K_{\max }=1$ & $K_{\max }=1$ \\
$\left.\leq 61314.24 \mathrm{~mm}^{3}\right)$ & $g=0.9$ & $g=0.5$ \\
& $d=0.01$ & $d=0.2$ \\
\hline & $b=0.2$ & $b=20$ \\
Size 4 & $k=0.9$ & $k=0.9$ \\
$\left(V>61314.24 \mathrm{~mm}^{3}\right)$ & $K_{\max }=1$ & $K_{\max }=1$ \\
& $g=0.9$ & $g=0.1$ \\
& $d=0.1$ & $d=0.01$ \\
\hline
\end{tabular}

Table 2: The values of treatment-specific parameters fitted using grid search for the case of chemotherapy (left) and immunotherapy (right). 


\subsection{Experiments}

\section{Comparing the fit of the game-theoretic model to that classical model}

In experiments, we evaluated how well the game-theoretical model defined by equations (1) -(2) matches the tumor volume trajectories of patients subject to immunotherapy or chemotherapy and compared the results to the ones obtained with the classical ODE models explored in [12]. In order to compare the performance between the game-theoretic model and the six models explored in [12, we calculated different metrics of the goodness of fit (for each of the study arms and each of the trend categories), namely the R2-score and the root mean squared error (RMSE). Further, we compare the fit of the model with and without evolution of resistance to see whether a possible better fit is due to more parameters in the game-theoretic model or rather due to its assumption on therapy resistance.

\section{Predicting treatment response based on initial data points only}

After fitting values of the treatment-specific parameters of each group, we test whether the model can predict the last data points from the early treatment response. More specifically, if we have $n$ data points, we use the first $n-3$ points to estimate the values of the patientspecific parameters, $r, u(0)$ and $\sigma$, and we solve equations (1) and (2) to get the value of $x$ at the time of the last measurement. Then, we evaluate the result by computing the mean absolute error between the predicted volume at time $t_{n}$ and the real value.

\section{Optimizing a pre-defined treatment objective}

Here we expand our model into a Stackelberg evolutionary game [18, 22, 20, 19] where the physician as a rational leader tries to optimize the predefined criterium, here final tumor burden, by changing the timing and in case of chemotherapy also dosing of treatment the patient received. Different objective functions can be explored, such as minimizing the final tumor burden, minimizing the tumor burden at every time point, minimizing the variance of the volume of the tumor, etc.

We consider the objective of minimizing the final tumor burden, which corresponds to solving the following optimization problem:

$$
\begin{aligned}
m^{*}(\cdot) & =\arg \min _{m(t)} x(T) \\
\dot{x}(t) & =x(t) G(m(t), u(t), x(t)) \\
\dot{u}(t) & =\sigma \frac{\partial G(m(t), u(t), x(t))}{\partial u(t)} \\
G(m(t), u(t), x(t)) & =r\left(1-\frac{x(t)}{K(u(t))}\right)-\frac{m(t)}{k+b u(t)}-d, \quad t \in[0, T],
\end{aligned}
$$

where $T$ is the final point considered. For patients subject to immunotherapy, we assume that we can decide whether treatment is on or off at every time point where we have a measurement, and so, at each of the time points, the optimal value of $m \in\{0,1\}$ is computed. On the other hand, for patients that received chemotherapy, we consider $m \in[0,1]$ and compute the optimal dose at each of the time points. 


\section{Simulating a different treatment scenario}

Since we have assumed that there is a set of parameters that are treatment-specific, namely $K_{\max }, b, d, g$ and $k$, we can use them to simulate what would have happened if the patients who received immunotherapy were being given chemotherapy and vice versa.

To do so, we assume that the initial value of the resistance to treatment is the same for both treatments. We take the parameters $K_{\max }, b, k, g$ and $d$ estimated for the corresponding treatment and the parameters $u(0), r_{\max }, \sigma$ estimated for the corresponding patient, and we simulate the evolution of the tumor subject to this treatment.

Thus, for patients who received Docetaxel, we simulated what would have happened if they had received immunotherapy, and vice versa.

\section{Simulating a treatment targeting the evolution of resistance}

Here we assume there exists a treatment that targets evolution of resistance, that is, that makes $u$ decrease instead of increase, and explore what would be its effect on the tumor evolution.

\section{Results}

\subsection{Comparing the fit of the game-theoretic model with classical ODE models}

As shown in Figures 1 and 2, in general the game-theoretic model obtains better scores for both metrics in most of the groups, although the difference of performance is not as significant in every trend category. Indeed, for the categories "Up", "Down" and "Fluctuate", the R2-score values are often not far from the best scores obtained with the other models, while for the tumors in the category "Evolution", the game-theoretic model significantly outperforms the other models in most of the arms. In fact, with the game-theoretic model we obtain an R2 score higher than 0.8 for all arms except one, compared to values below 0.6 with the classic ODE models in most of the arms. This indicates that the game-theoretic model is indeed more suitable to describe tumors that exhibit an evolution of resistance to treatment, featuring a U-shape in their trend of growth over time. Figure 3 shows two fits obtained for tumors in the category "Evolution", that could not be well fitted with classical ODE models.

On the other hand, the R2-score for tumors in the trend category "Fluctuate" is below 0.6 in most of the groups for all of the models, which indicates a systematic limitation to accurately fit tumors that experience pseudoprogression, i.e., tumors that keep growing for a relatively long period of time after the start of treatment and then start responding to treatment, or tumors that grow and decrease alternatively over time.

According to our results, the fits given by the game-theoretic model are generally better than those of classical models. In order to determine whether this superiority is purely due to the higher complexity of the model or also influenced by the assumption of including resistance as an evolving trait, we compared the results obtained with the game-theoretic model when we assume there is no evolution of resistance $(\sigma=0)$ with those obtained when we assume that evolution of resistance occurs $(\sigma>0)$. 
Figure 4 shows that, generally, tumors that exhibit a monotonic behaviour, are fitted well under the assumption that resistance does not evolve. However, tumors that exhibit changing trends over time - typically presented as initial response to treatment followed by an increase of volume over time - cannot be well fitted if we set $\sigma=0$ (Figure 5). These cases support the importance of modeling resistance as an evolving trait.

\subsection{Predicting treatment response based on initial data points}

In general, we obtain a lower mean absolute error with the game-theoretic model than with the ones used in [12] (Figure 6). Of particular interest is the case of U-shaped tumors, that could not be well predicted by any of the classic ODE models explored in [12]. For most of these tumors, the game-theoretic model provides a more accurate prediction (Figure 7 shows two examples), although in some cases the estimated growth due to evolution of resistance is considerably higher than the real growth, while in some other cases, where resistance appears at a later time the model fails to predict this growth.

\subsection{Optimizing a pre-defined treatment objective}

Simulations show that in general it is not possible to stabilize the tumor volume, and that the optimal strategy is usually to treat patients continuously at MTD.

This is partially due to our objective being the final tumor burden: for a limited number of patients we obtain that a different treatment schedule leads to the same final tumor burden. For example, Figure 8 shows the case of two patients who received Docetaxel, where lowering the dose of treatment yields the same final tumor burden reached by applying constant MTD, although the tumor volume at previous time points is higher. The interest of this result lies in the fact that lower treatment dose often corresponds to higher quality of life, as the side effects and discomfort caused by the medication are reduced.

Furthermore, if we simulate the evolution of the tumor for a longer period of time aiming at minimizing the tumor burden at the final time point, we obtain that for a limited number of cases allowing the tumor to grow at the beginning results in a lower final tumor burden (Figure 9). Nevertheless, when following this treatment schedule, the tumor tends to grow until it has reached a volume close to the maximum over all the patients of the dataset.

The reason why it is hard to design an evolutionary treatment for these patients is that the growth rate of stage 4 NSCLC tumors is too high to allow the tumor to grow unrestricted for some periods of time [ 8 , 7].

On the other hand, the treatment objective here targets the tumor burden only, thus it may be that optimizing a proper quality of life measure will lead to more satisfactory results and an evolutionary therapy that successfully anticipates and steers therapy resistance.

\subsection{Simulating a different treatment scenario}

For patients who received Docetaxel, we simulated what would have happened if they had received immunotherapy, and we obtained that the majority were better off with the given treatment: $75 \%$ of the patients obtained a lower final tumor burden with Docetaxel than with immunotherapy. Figure 10 shows the comparison between the real tumor evolution of a patient subject to Docetaxel and the simulation of the evolution under immunotherapy, which would lead to a noticeably higher final tumor burden. 
On the other hand, when we simulated the behaviour under Docetaxel for patients who were treated with immunotherapy, the percentage of patients that obtained a lower final tumor burden with the given treatment dropped to $50 \%$. Figure 11 shows an example of a tumor that would have responded better to Docetaxel than immunotherapy according to simulations.

It is important to note that these results are heavily based on the assumptions that the parameters estimated are treatment-specific and that the initial resistance to both treatments is the same.

A thorough validation of these parameters is needed in order to predict beforehand which treatment would be optimal for each patient.

\subsection{Impact of a treatment targeting the evolution of resistance}

If we assume the existence of a treatment that targets evolution of resistance, we obtain that $58 \%$ of the patients would achieve a lower tumor burden than with the treatment administered in reality (Figure 12 reports two such examples). Moreover, the percentage of tumors such that the final tumor burden is lower than the starting one is $75 \%$, compared to $63 \%$ with the existing treatments. This alternative treatment is especially useful for tumors in the trend categories "Up" and "Evolution": more than $70 \%$ of the tumors of these categories respond better to the alternative treatment than to traditional treatments, meaning that the final tumor burden is smaller.

\section{Discussion}

We have shown that metastatic non-small cell lung cancer data are more accurately fitted with game-theoretical models which allows for the evolution of therapy resistance than with classic ODE models. Especially tumors which exhibit a U-shape in their trend were fitted well by the game-theoretical models, while such trends could not be fitted at all with previous models, as stated in [12].

Furthermore, modeling resistance as an evolutionary trait has been shown to have a relevant impact on the results and thus, these types of models should be applied more often to further explore their relevance to clinical outcomes across many different cancer types.

Moreover, predicting the trend of the tumor given its initial response with the model proposed in this paper also yields a lower error, although the results obtained show that in some cases predictions are still not satisfactory. More accurate predictions could be achieved by reducing the number of patient-specific parameters or by providing more information about their values, i.e., setting stricter boundaries for the values of the parameters or starting with better estimates of the initial value of $u$, the growth rate $r$ and the evolutionary speed $\sigma$. On the other hand, designing evolutionary therapies with such a restricted amount of information on tumor treatment response proved to be a challenging task. With the model presented here, stabilizing the tumors is not feasible, but it is possible to slow down their growth in some cases, especially for tumors that start growing exponentially from the beginning or after a period of response to treatment. The reason for these impediments is that the growth rate of these tumors is too high to let them grow unrestricted for longer periods of time. Therefore, in this type of cancer, treating continuously at MTD often seems to yield to a better outcome than adaptive therapy, which implies that in order to improve the life 
expectancy/quality of the patients, alternative treatments, different measures of treatment success, or more a priori information concerning the effect that the available treatments will have on the growth of the tumors are needed.

That is why simulation of tumor growth under different treatments is a task of interest. We have simulated the evolution of the tumors as if they had been subject to immunotherapy for chemotherapy patients and vice-versa. It is important to note that these results are heavily based on the assumptions that the parameters estimated are characteristic of the behaviour of tumors under the specific treatment and that the initial resistance to both treatments are the same. Validation of these simulations would allow us to be able to decide which treatment is better for each patient beforehand furthering precision medicine in cancer significantly. Finally, simulations exploring what would happen if there existed a treatment that targets the evolution of resistance were also undertaken, showing that this could improve the final outcome for more than half of the patients, especially those who don't respond to treatment due to the evolution of resistance. Nevertheless, actual evidence of the existence of such treatments is still quite preliminary.

Better knowledge regarding resistance mechanisms in the case of immunotherapy could help us to adapt the model in order to make it more faithful to reality. On the other hand, having real estimates of some of the parameters based on experimentation - especially of the growth and death rates of cancer cells - or more restricted bounds of the exploration range of the parameters could lead to more realistic simulations, and could improve our prediction and optimization results.

Additionally, the model shows a systematic failure to fit cases of tumors which experience pseudoprogression, i.e., tumors that keep growing for a relatively long period of time after the start of treatment and then start responding to treatment. This is one of the aspects where clear improvement is needed, as this delayed response to immunotherapy is a phenomenon that is of great interest for physicians - although their main goal is to predict when this will happen. This prediction might not necessarily be possible with an evolutionary model alone (at the very least some additional biomarkers are probably needed). On the methodological side, delays can be incorporated into the ecological dynamics model which could capture this delayed response to treatment.

Future research should focus on modelling the so-called pseudoprogression response in immunotherapy and on obtaining more medical information, namely estimates for the parameters and, in particular, for the resistance $u$, which would lead to more realistic simulations and could help validate the results obtained here.

\section{Acknowledgements}

The authors thank F. Hoffmann-La Roche Ltd. for sharing the raw data through the platform "Clinical Study Data Request" (CSDR, www. ClinicalStudyDataRequest.com). This research was supported by European Union's Horizon 2020 research and innovation programs under the Marie Skłodowska-Curie grants 690817 and 955708, the Dutch National Foundation projects ENWPR.020.006 and OCENW.KLEIN.277. 


\section{Disclosures}

JNK declares consulting services for Owkin, France and Panakeia, UK. No other potential conflicts of interest are reported by any of the authors.

\section{References}

[1] Robyn P Araujo and DL Sean McElwain. A history of the study of solid tumour growth: the contribution of mathematical modelling. Bulletin of mathematical biology, 66(5):1039-1091, 2004.

[2] Logan D. R. Beal, Daniel C. Hill, R. Abraham Martin, and John D. Hedengren. Gekko optimization suite. Processes, 6(8), 2018. doi:10.3390/pr6080106.

[3] Ranjini Bhattacharya, Robert Vander Velde, Viktoriya Marusyk, Bina Desai, Artem Kaznatcheev, Andriy Marusyk, and David Basanta. Understanding the evolutionary games in NSCLC microenvironment. bioRxiv, 2020. doi:10.1101/2020.11.30. 404350 .

[4] Cancer.Net. Lung cancer - Non-Small Cell: Statistics, 2020.

[5] Yosef Cohen, Thomas Vincent, and Joel S Brown. A G-function approach to fitness minima, fitness maxima, evolutionary stable strategies and adaptive landscapes. Evolutionary Ecology Research, 1, 121999.

[6] Jessica Cunningham, Frank Thuijsman, Ralf Peeters, Yannick Viossat, Joel Brown, Robert Gatenby, and Kateřina Staňková. Optimal control to reach eco-evolutionary stability in metastatic castrate-resistant prostate cancer. Plos One, 15(12):e0243386, 2020 .

[7] Dirk De Ruysscher, José Belderbos, Bart Reymen, Wouter van Elmpt, Angela van Baardwijk, Rinus Wanders, Frank Hoebers, Marc Vooijs, Michel Öllers, and Philippe Lambin. State of the art radiation therapy for lung cancer 2012: A glimpse of the future. Clinical Lung Cancer, 14(2):89-95, 2013.

[8] Dirk De Ruysscher, Angela van Baardwijk, Rinus Wanders, Lizza E. Hendriks, Bart Reymen, Wouter van Empt, Michel C. Öllers, Gerben Bootsma, Cordula Pitz, Linda van Eijsden, and Anne-Marie C. Dingemans. Individualized accelerated isotoxic concurrent chemo-radiotherapy for stage III non-small cell lung cancer: 5-year results of a prospective study. Radiotherapy and Oncology, 135:141-146, 2019.

[9] Ana Faustino-Rocha, Paula A Oliveira, Jacinta Pinho-Oliveira, Catarina TeixeiraGuedes, Ruben Soares-Maia, Rui Gil Da Costa, Bruno Colaco, Maria Joao Pires, Jorge Colaco, Rita Ferreira, et al. Estimation of rat mammary tumor volume using caliper and ultrasonography measurements. Lab animal, 42(6):217-224, 2013.

[10] Robert A Gatenby. A change of strategy in the war on cancer. Nature, 459(7246):508509, 2009. 
[11] Robert A Gatenby, Ariosto S Silva, Robert J Gillies, and B Roy Frieden. Adaptive therapy. Cancer research, 69(11):4894-4903, 2009.

[12] Narmin Ghaffari Laleh, Chiara Maria Lavinia Loeffler, Julia Grajek, Kateřina Staňková, Alexander T. Pearson, Hannah Sophie Muti, Christian Trautwein, Heiko Enderling, Jan Poleszuk, and Jakob Nikolas Kather. Classical mathematical models for prediction of response to chemotherapy and immunotherapy. bioRxiv. doi:10.1101/2021.10. 23.465549 .

[13] Ahmedin Jemal, Elizabeth M Ward, Christopher J Johnson, Kathleen A Cronin, Jiemin Ma, A Blythe Ryerson, Angela Mariotto, Andrew J Lake, Reda Wilson, Recinda L Sherman, et al. Annual report to the nation on the status of cancer, 1975-2014, featuring survival. JNCI: Journal of the National Cancer Institute, 109(9):djx030, 2017.

[14] Artem Kaznatcheev, Jeffrey Peacock, David Basanta, Andriy Marusyk, and Jacob G Scott. Fibroblasts and alectinib switch the evolutionary games played by non-small cell lung cancer. Nature ecology \& evolution, 3(3):450-456, 2019.

[15] Félicien Le Louedec, Fanny Leenhardt, Clémence Marin, Étienne Chatelut, Alexandre Evrard, and Joseph Ciccolini. Cancer immunotherapy dosing: A pharmacokinetic/pharmacodynamic perspective. Vaccines, 8(4):632-642, 2020.

[16] Mariyah Pressley, Monica Salvioli, David B Lewis, Christina L Richards, Joel S Brown, and Kateřina Staňková. Evolutionary dynamics of treatment-induced resistance in cancer informs understanding of rapid evolution in natural systems. Frontiers in Ecology and Evolution, 9:460, 2021.

[17] Monica Salvioli. Game theory for improving medical decisions and managing biological systems. PhD thesis, Italy, 2020.

[18] Monica Salvioli, Johan Dubbeldam, Kateřina Staňková, and Joel S Brown. Fisheries management as a Stackelberg evolutionary game: Finding an evolutionarily enlightened strategy. Plos One, 16(1):e0245255, 2021.

[19] Monica Salvioli, Hasti Garjani, Joel S. Brown, Johan Dubbeldam, and Kateřina Staňková. Stackelberg evolutionary games of cancer treatment: Tumor stabilization as an alternative to dynamic treatment protocols. Under review, 2021.

[20] K. Staňková. Resistance games. Nature Ecology 8 Evolution, 3:336-337, 2019. doi: $10.1038 / \mathrm{s} 41559-018-0785-\mathrm{y}$.

[21] Thomas L Vincent and Joel S Brown. Evolutionary game theory, natural selection, and Darwinian dynamics. Cambridge University Press, 2005.

[22] Benjamin Wölfl, Hedy te Rietmole, Monica Salvioli, Artem Kaznatcheev, Frank Thuijsman, Joel S Brown, Boudewijn Burgering, and Kateřina Staňková. The contribution of evolutionary game theory to understanding and treating cancer. Dynamic Games and Applications, pages 1-30, 2021. 
[23] Jingsong Zhang, Jessica J Cunningham, Joel S Brown, and Robert A Gatenby. Integrating evolutionary dynamics into treatment of metastatic castrate-resistant prostate cancer. Nature communications, 8(1):1-9, 2017. 
bioRxiv preprint doi: https://doi.org/10.1101/2021.10.29.466444; this version posted November 1, 2021. The copyright holder for this preprint (which was not certified by peer review) is the author/funder. All rights reserved. No reuse allowed without permission.

\begin{tabular}{|c|c|c|c|c|c|c|c|c|}
\hline \multirow[b]{2}{*}{ MPOL3280A_1_Up } & \multicolumn{7}{|c|}{ R-squared values for each arm } & 10 \\
\hline & 0.94 & 0.94 & 0.99 & 0.94 & 0.94 & 0.94 & 0.97 & -10 \\
\hline MPDL3280A_1_Down & 0.91 & 0.93 & 0.92 & 0.94 & 0.91 & 0.94 & 0.97 & \\
\hline MPDL_3280A_1_Fluctuate & 0.59 & 0.51 & 0.59 & 0.59 & 0.59 & 0.6 & 0.54 & \\
\hline MPDL_3280A_1_Evolution & 0.28 & 0.13 & 0.47 & 0.41 & 0.3 & 0.3 & 0.81 & \\
\hline MPDL3280A_2_Up & 0.54 & 0.86 & 0.47 & 0.85 & 0.85 & 0.85 & 0.96 & \\
\hline MPDL3280A_2_Down & 0.89 & 0.91 & 0.89 & 0.93 & 0.93 & 0.92 & 0.98 & \\
\hline MPDL3280A_2_Fluctuate & 0.57 & 0.55 & 0.64 & 0.6 & 0.59 & 0.61 & 0.6 & \\
\hline MPDL_3280A_2_Evolution & 0.54 & 0.65 & 0.72 & 0.66 & 0.66 & 0.66 & 0.96 & \\
\hline MPDL3280A_3_Down & 0.9 & 0.96 & 0.9 & 0.97 & 0.97 & 0.97 & 0.94 & \\
\hline DOCETAXEL_Down & 0.78 & 0.84 & 0.83 & 0.85 & 0.84 & 0.81 & 0.9 & -0.8 \\
\hline DOCETAXEL_Fluctuate & 0.45 & 0.61 & 0.46 & 0.63 & 0.63 & 0.63 & 0.68 & \\
\hline DOCETAXEL_Evolution & 0.27 & 0.4 & 0.53 & 0.3 & 0.3 & 0.3 & 0.93 & \\
\hline MPDL3280A_Up & 0.81 & 0.81 & 0.72 & 0.81 & 0.81 & 0.81 & 0.79 & \\
\hline MPDL_3280A_Down & 0.78 & 0.84 & 0.79 & 0.88 & 0.87 & 0.88 & 0.89 & \\
\hline MPDL.3280A_Fluctuate & 0.52 & 0.54 & 0.56 & 0.53 & 0.53 & 0.54 & 0.61 & \\
\hline MPDL3280A_Evolution & 0.47 & 0.44 & 0.66 & 0.52 & 0.52 & 0.53 & 0.88 & \\
\hline Cohort 1a (Squamous)_Down & 0.94 & 0.95 & 0.96 & 0.95 & 0.97 & 0.98 & 0.96 & \\
\hline Cohort 1a (Squamous)_Fluctuate & 0.6 & 0.6 & 0.69 & 0.6 & 0.6 & 0.63 & 0.76 & \\
\hline Cohort $1 \mathbf{a}$ (Squamous)_Evolution & 0.84 & 0.84 & 0.96 & 0.84 & 0.84 & 0.84 & 0.97 & -0.6 \\
\hline Cohort ib (Non-Squamous)_Up & 0.93 & 0.93 & 0.97 & 0.93 & 0.93 & 0.93 & 0.85 & \\
\hline Cohort 1b (Non-Squamous)_Down & 0.85 & 0.88 & 0.85 & 0.93 & 0.92 & 0.92 & 0.95 & \\
\hline Cohort ib (Non-Squamous)_Fluctuate & 0.34 & 0.42 & 0.36 & 0.43 & 0.41 & 0.43 & 0.34 & \\
\hline Cohort ib (Non-Squamous)_Evolution & 0.49 & 0.49 & 0.29 & 0.49 & 0.49 & 0.49 & 0.81 & \\
\hline Cohort 2a (Squamous)_Up & 0.73 & 0.88 & 0.74 & 0.88 & 0.88 & 0.88 & 0.81 & \\
\hline Cohort 2a (Squamous)_Down & 0.85 & 0.92 & 0.87 & 0.95 & 0.95 & 0.95 & 0.96 & \\
\hline Cohort 2a (Squamous)_Fluctuate & 0.48 & 0.36 & 0.66 & 0.5 & 0.48 & 0.49 & 0.61 & \\
\hline Cohort 2a (Squamous)_Evolution & 0.32 & 0.32 & 0.68 & 0.32 & 0.32 & 0.32 & 0.92 & \\
\hline Cohort 2b (Non-Squamous)_Up & 0.83 & 0.84 & 0.91 & 0.84 & 0.84 & 0.84 & 0.89 & \\
\hline Cohort 2b (Non-Squamous)_Down & 0.84 & 0.88 & 0.86 & 0.9 & 0.88 & 0.88 & 0.96 & 4 \\
\hline Cohort 2b (Non-Squamous)_Fluctuate & 0.38 & 0.43 & 0.36 & 0.46 & 0.46 & 0.46 & 0.23 & \\
\hline Cohort 2b (Non-Squamous)_Evolution & 0.53 & 0.54 & 0.67 & 0.6 & 0.6 & 0.6 & 0.97 & \\
\hline Cohort 3a (Squamous)_Up & 0.78 & 0.82 & 0.86 & 0.82 & 0.82 & 0.82 & 0.33 & \\
\hline Cohort 3s (Squamous)_Down & 0.99 & 0.99 & 0.99 & 0.99 & 0.99 & 0.99 & 1 & \\
\hline Cohort 3a (Squamous)_Fluctuate & 0.17 & 0.17 & 0.24 & 0.18 & 0.18 & 0.18 & 0.038 & \\
\hline Cohort 3a (Squamous)_Evolution & 0.33 & 0.47 & 0.32 & 0.47 & 0.47 & 0.47 & 0.26 & \\
\hline Cohort 3b (Non-Squamous)_Up & 0.73 & 0.82 & 0.71 & 0.82 & 0.82 & 0.82 & 0.56 & \\
\hline Cohort 3b (Non-Squamous)_Down & 0.9 & 0.76 & 0.92 & 0.91 & 0.91 & 0.95 & 0.96 & \\
\hline Cohort 3b (Non-Squamous)_Fluctuate & 0.47 & 0.44 & 0.51 & 0.51 & 0.5 & 0.51 & 0.62 & -0.2 \\
\hline Cohort 3b (Non-Squamous)_Evolution & 0.51 & 0.67 & 0.57 & 0.67 & 0.67 & 0.67 & 0.81 & \\
\hline Docataxe__Up & 0.71 & 0.85 & 0.7 & 0.85 & 0.85 & 0.85 & 0.56 & \\
\hline Docataxe|_Down & 0.67 & 0.83 & 0.69 & 0.88 & 0.88 & 0.89 & 0.95 & \\
\hline Docetaxel_Fluctuate & 0.29 & 0.39 & 0.34 & 0.39 & 0.39 & 0.41 & 0.34 & \\
\hline Docetaxel_Evolution & 0.3 & 0.4 & 0.4 & 0.48 & 0.46 & 0.46 & 0.91 & \\
\hline MPDL3280A_Up & 0.73 & 0.81 & 0.71 & 0.81 & 0.81 & 0.81 & 0.78 & \\
\hline MPOL3280A_Down & 0.83 & 0.9 & 0.84 & 0.93 & 0.92 & 0.93 & 0.95 & \\
\hline MPDL3280A_Fluctuate & 0.34 & 0.42 & 0.39 & 0.43 & 0.42 & 0.42 & 0.52 & \\
\hline MPDL3280A_Evolution & 0.53 & 0.5 & 0.68 & 0.56 & 0.57 & 0.57 & 0.9 & \\
\hline
\end{tabular}

Figure 1: R2 score values for each model and each trend category, where each model was fitted separately for that category. A higher value corresponds to a better fit. 
bioRxiv preprint doi: https://doi.org/10.1101/2021.10.29.466444; this version posted November 1, 2021. The copyright holder for this preprint (which was not certified by peer review) is the author/funder. All rights reserved. No reuse allowed without permission.

\begin{tabular}{|c|c|c|c|c|c|c|c|c|}
\hline & & & & & & & & -0.005 \\
\hline MPDL3280A_1_Up & 0.016 & 0.00025 & 0.00013 & 0.00025 & 0.00025 & 0.00025 & 0.0002 & \\
\hline MPDL3280A_1_Down & 0.027 & 0.0022 & 0.0021 & 0.0021 & 0.0021 & 0.0021 & 7.1e-05 & \\
\hline MPOL3280A_1_Fluctuste & 0.0026 & 7.7e-05 & $6.3 e-05$ & $6.3 e-05$ & 6.3e-05 & $6.3 \mathrm{e}-05$ & $7.1 \mathrm{e}-05$ & \\
\hline MPDL3280A_1_Evolution & 0.025 & 0.002 & 0.0019 & 0.002 & 0.0024 & 0.0024 & 0.0009 & \\
\hline MPDL3280A_2_Up & 0.027 & 0.0044 & 0.029 & 0.0036 & 0.0036 & 0.0036 & 0.0011 & \\
\hline MPDL3280A_2_Down & 0.013 & 0.0029 & 0.0018 & 0.0017 & 0.0017 & 0.0018 & 0.00013 & \\
\hline MPOL3280A_2_Fluctuate & 0.026 & 0.0025 & 0.0023 & 0.0023 & 0.0023 & 0.0022 & 0.0013 & \\
\hline MPDL3280A_2_Evolution & 0.043 & 0.0057 & 0.0047 & 0.0057 & 0.0057 & 0.0057 & 0.0012 & \\
\hline MPOL_3280A_3_Down & 0.0048 & 0.00028 & 0.0006 & 0.0003 & 0.0003 & 0.0003 & $3.2 \mathrm{e}-05$ & \\
\hline DOCETAXEL_Down & 0.065 & 0.0042 & 0.0048 & 0.0041 & 0.0041 & 0.0041 & 7.1e-05 & -0.004 \\
\hline DQCETAXEL_Fluctuat: & 0.37 & 0.0042 & 0.004 & 0.0042 & 0.0042 & 0.0042 & 0.00026 & \\
\hline DOCETAXEL_Evolution & 0.16 & 0.0017 & 0.0015 & 0.0018 & 0.0018 & 0.0018 & 0.00025 & \\
\hline MPDL3280A_Up & 0.36 & 0.0033 & 0.0056 & 0.0034 & 0.0034 & 0.0034 & 0.00072 & \\
\hline MPDL3280A_Down & 0.23 & 0.0032 & 0.0076 & 0.0034 & 0.0034 & 0.0034 & 0.00047 & \\
\hline MPDL3280A_Fluctuate & 0.3 & 0.0048 & 0.0047 & 0.0046 & 0.0046 & 0.0046 & 0.0013 & \\
\hline MPDL3280A_Evolution & 0.36 & 0.0049 & 0.0028 & 0.0046 & 0.0046 & 0.0046 & 0.00095 & \\
\hline Cohort 1a (Squamous)_Down & 0.011 & 0.00049 & 0.00048 & 0.00032 & 0.00032 & 0.00027 & 0.00017 & \\
\hline Cohort 1a (Squamous)__Fluctuate & 0.034 & 0.0029 & 0.0011 & 0.0023 & 0.0023 & 0.002 & 0.00043 & \\
\hline Cohort 1a (Squamous)_Evolution & 0.0032 & 0.00019 & 0.0001 & 0.00019 & 0.00019 & 0.00019 & $8.9 e-05$ & -0.003 \\
\hline Cohort ib (Non-Squamous)_Up & 0.013 & 0.0009 & 0.00072 & 0.0009 & 0.0009 & 0.0009 & 0.00068 & \\
\hline Cohort $1 \mathrm{~b}$ (Non-Squamous)_Down & 0.011 & 0.00051 & 0.00062 & 0.00049 & 0.00049 & 0.00048 & $7.7 e-05$ & \\
\hline Cohort to (Non-Squamous)_Fluctuate & 0.019 & 0.00048 & 0.00047 & 0.00047 & 0.00047 & 0.00047 & 0.00026 & \\
\hline Cohort ib (Non-Squamous)_Evolution & 0.0067 & 0.00028 & 0.00024 & 0.00028 & 0.00028 & 0.00028 & 0.00018 & \\
\hline Cohort 2a (Squamous)_Up & 0.042 & 0.0031 & 0.0026 & 0.0031 & 0.0031 & 0.0031 & 0.0022 & \\
\hline Cohort 2a (Squamous)_Down & 0.014 & 0.00042 & 0.00082 & 0.00033 & 0.00033 & 0.00034 & 0.00013 & \\
\hline Cohort 2a (Squamous)_Fluctuate & 0.048 & 0.0032 & 0.0022 & 0.0031 & 0.0031 & 0.0031 & 0.002 & \\
\hline Cohort 2a (Squamous)_Evolution & 0.013 & 0.0017 & 0.0012 & 0.0017 & 0.0017 & 0.0017 & 0.00058 & \\
\hline Cohort $2 \mathrm{~b}$ (Non-Squamous)_Up & 0.024 & 0.0017 & 0.0012 & 0.0017 & 0.0017 & 0.0017 & 0.00025 & \\
\hline Cohort 2b (Non-Squamous)_Down & 0.032 & 0.0029 & 0.0028 & 0.0027 & 0.0027 & 0.0028 & 0.00017 & -0.002 \\
\hline Cohort 2b (Non-Squamous)_Fluctuate & 0.019 & 0.0011 & 0.00077 & 0.0012 & 0.0012 & 0.0012 & 0.0002 & \\
\hline Cohort $2 b$ (Non-Squamous)_Evolution & 0.009 & 0.0026 & 0.0021 & 0.0025 & 0.0025 & 0.0025 & 0.00011 & \\
\hline Cohort 3a (Squamous)_Up & 0.034 & 0.0015 & 0.00085 & 0.0015 & 0.0015 & 0.0015 & 0.00071 & \\
\hline Cohort 3a (Squamous)_Down & 0.011 & 0.00056 & 0.00046 & 0.00022 & 0.00025 & 0.00017 & $9.5 e-05$ & \\
\hline Cohort 3a (Squamous)_Fluctuate & 0.071 & 0.0054 & 0.0049 & 0.0054 & 0.0054 & 0.0054 & 0.0027 & \\
\hline Cohort 3a (Squamous)_Evolution & 0.063 & 0.002 & 0.0021 & 0.002 & 0.002 & 0.002 & 0.002 & \\
\hline Cohort 3b (Non-Squamous)_Up & 0.071 & 0.00084 & 0.0018 & 0.00087 & 0.00086 & 0.00086 & 0.00083 & \\
\hline Cohort 3b (Non-Squamous)_Down & 0.03 & 0.0042 & 0.0028 & 0.0034 & 0.0034 & 0.0033 & $3.2 \mathrm{e}-05$ & \\
\hline Cohort 3b (Non-Squamous)_Fluetuate & 0.024 & 0.0026 & 0.0024 & 0.0026 & 0.0026 & 0.0026 & 0.00044 & -0.001 \\
\hline Cohort 3b (Non-Squamous)_Evolution & 0.022 & 0.00072 & 0.0014 & 0.00072 & 0.00072 & 0.00071 & 0.00052 & \\
\hline Docetaxel_Up & 0.0014 & 0.001 & 0.0015 & 0.001 & 0.001 & 0.001 & 0.00032 & \\
\hline Docetaxel_Down & 0.0041 & 0.002 & 0.004 & 0.002 & 0.002 & 0.002 & 0.00023 & \\
\hline Doceisxel_Fluctuate & 0.0079 & 0.0065 & 0.0068 & 0.0073 & 0.0073 & 0.0073 & 0.00048 & \\
\hline Docataxel_Evolution & 0.0029 & 0.0028 & 0.0026 & 0.0027 & 0.0027 & 0.0027 & 0.00026 & \\
\hline MPDL3280A_UP & 0.0037 & 0.0028 & 0.0036 & 0.0028 & 0.0028 & 0.0028 & 0.00056 & \\
\hline MPDL3280A_Down & 0.02 & 0.021 & 0.016 & 0.02 & 0.02 & 0.021 & 0.00019 & \\
\hline MPDL3280A_Fluctuate & 0.0054 & 0.0043 & 0.0052 & 0.0043 & 0.0043 & 0.0043 & 0.0013 & \\
\hline MPDL3280A_Evolution & 0.0037 & 0.0039 & 0.0022 & 0.0037 & 0.0037 & 0.0037 & 0.00033 & -0.0 \\
\hline
\end{tabular}

Figure 2: Root mean squared error for each model and each trend category, where each model was fitted separately for that category. A lower value corresponds to a better fit. 

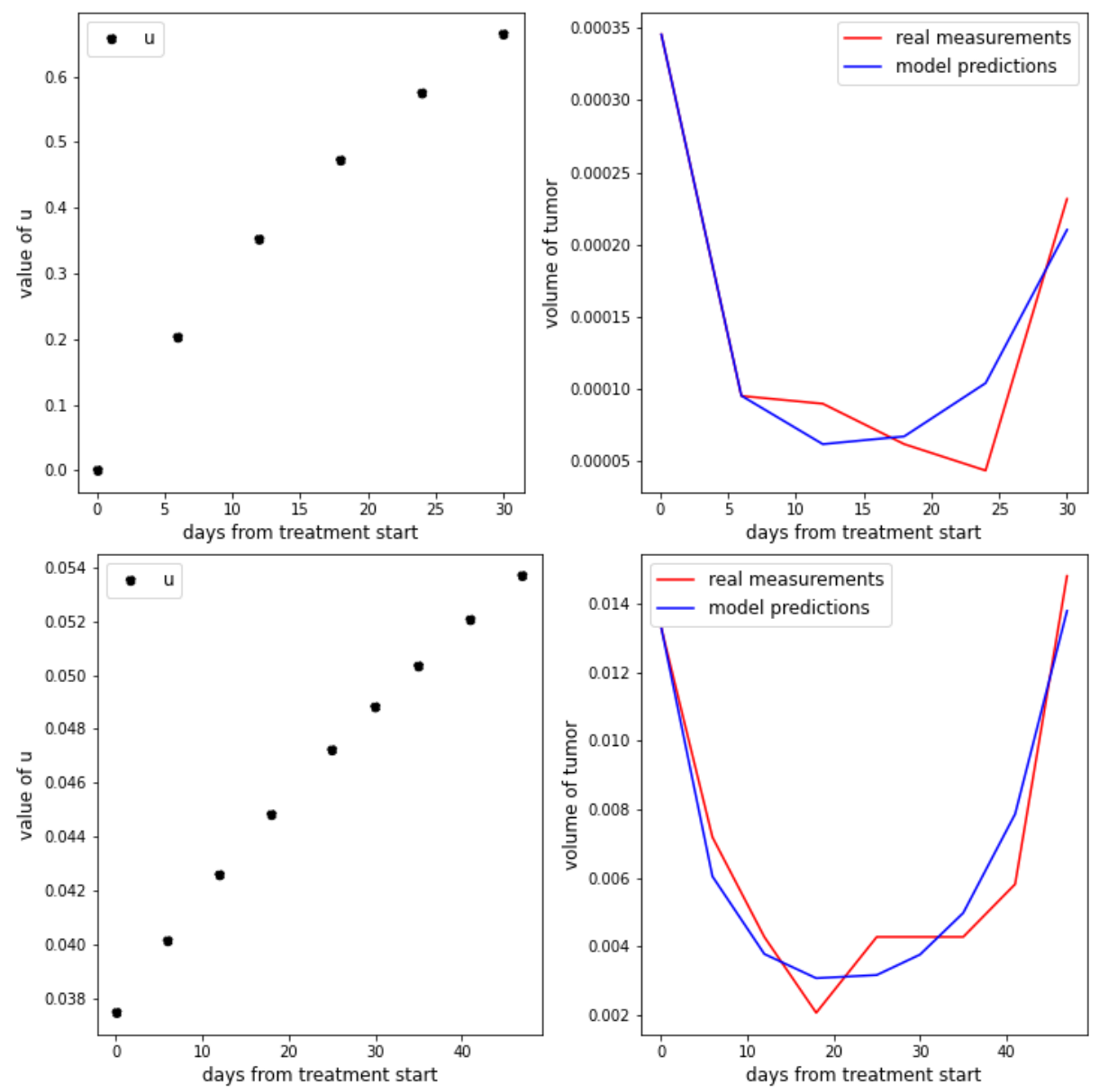

Figure 3: Two examples of the fits obtained for tumors in the "Evolution" category for a patient treated with Docetaxel (up) and a patient treated with immunotherapy (down).
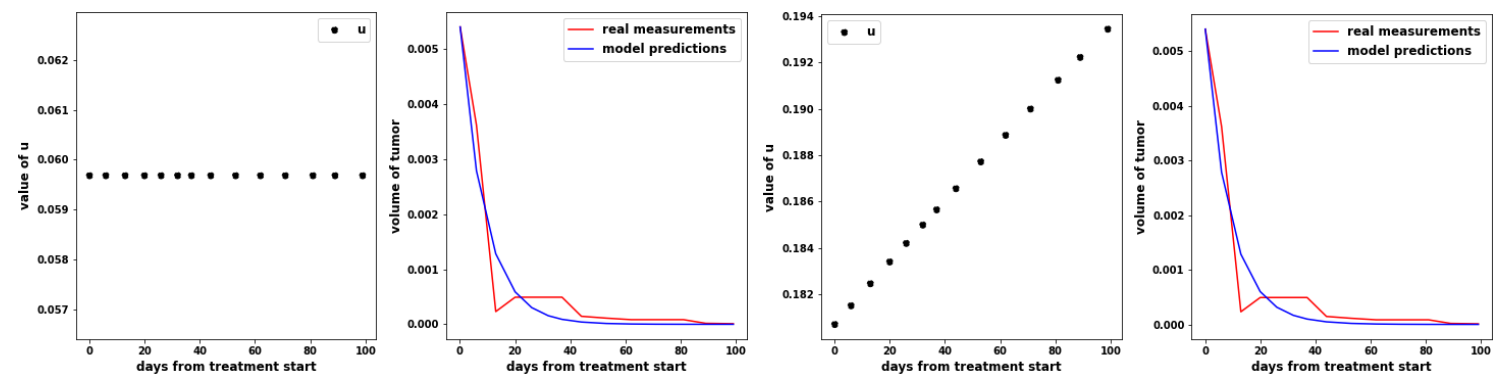

Figure 4: Fits obtained assuming there is no evolution of resistance (left) and assuming there is evolution of resistance (right) for a patient treated with immunotherapy. 

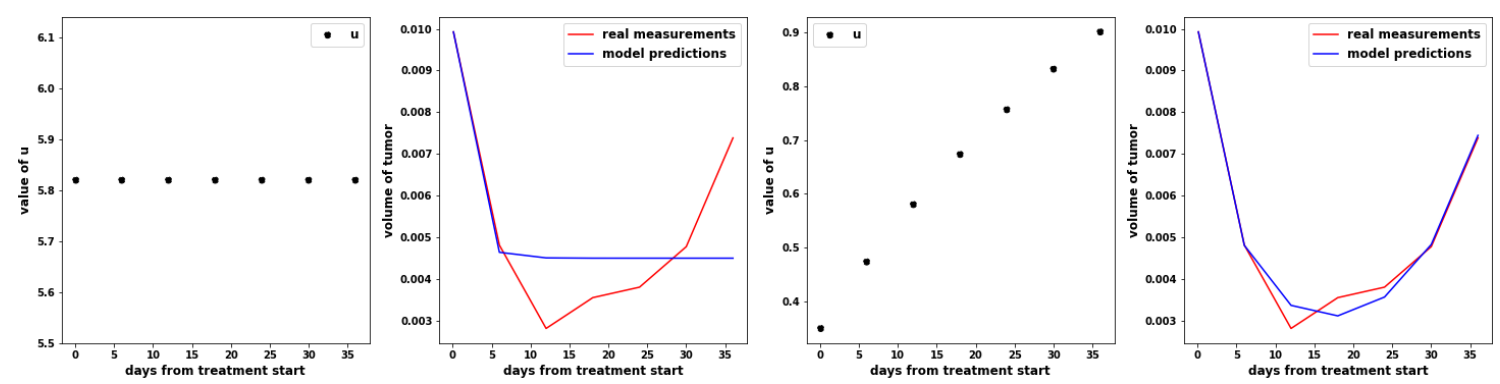

Figure 5: Fits obtained assuming there is no evolution of resistance (left) and assuming there is evolution of resistance (right) for a patient treated with Docetaxel. 
bioRxiv preprint doi: https://doi.org/10.1101/2021.10.29.466444; this version posted November 1, 2021. The copyright holder for this preprint (which was not certified by peer review) is the author/funder. All rights reserved. No reuse allowed without permission.

MAE values for each arm

\begin{tabular}{|c|c|c|c|c|c|c|c|c|}
\hline \multirow[b]{2}{*}{ MPOL3280A_1_Up } & & \multirow{2}{*}{-0.010} \\
\hline & 0.00031 & 0.00096 & 0.0051 & 0.00082 & 0.00053 & 0.0008 & 0.00041 & \\
\hline MPDL3280A_1_Down & 0.0011 & 0.0026 & 0.0011 & 0.0027 & 0.0027 & 0.0031 & 0.00079 & \\
\hline MPDL3280A_1_Fluctuate & $1.9 \mathrm{e}-05$ & $6.2 e-05$ & $3.4 \mathrm{e}-05$ & $1.9 e-05$ & $1.8 \mathrm{e}-05$ & 3.1e-05 & $7.4 e-05$ & \\
\hline MPDL3280A_1_Evolution & 0.006 & 0.005 & 0.006 & 0.0049 & 0.0049 & 0.0049 & 0.0032 & \\
\hline MPOL3280A_2_Up & 0.1 & 0.0029 & $1.5 e+06$ & 0.0063 & 0.0059 & 0.0063 & 0.0056 & \\
\hline MPDL3280A_2_Down & 0.00097 & 0.0017 & 0.0013 & 0.00068 & 0.00068 & 0.00098 & $8.7 e-05$ & \\
\hline MPDL3280A_2_Fluctuate & 0.0024 & 0.0031 & 0.002 & 0.0022 & 0.0023 & 0.0021 & 0.0018 & \\
\hline MPDL3280A_2_Evolution & 0.024 & 0.022 & 0.024 & 0.021 & 0.021 & 0.021 & 0.011 & \\
\hline MPDL3280A_3_Down & 0.00056 & 0.00017 & 0.00056 & $2.7 e-05$ & $4.7 \mathrm{e}-05$ & $2.8 \mathrm{e}-05$ & $6.1 e-05$ & \\
\hline DOCETAXEL_Down & 0.0013 & 0.0021 & 0.0013 & 0.0031 & 0.003 & 0.0032 & 0.00018 & -0.008 \\
\hline DOCETAXEL_Fluctuate & 0.0061 & 0.0047 & 0.19 & 0.005 & 0.0049 & 0.0049 & 0.018 & \\
\hline DOCETAXEL_Evolution & 0.0056 & 0.0041 & 0.0056 & 0.0049 & 0.0049 & 0.0049 & 0.0032 & \\
\hline MPDL3280A_Up & 0.0049 & 0.013 & $3.2 e+05$ & 0.013 & 0.013 & 0.013 & 0.0034 & \\
\hline MPDL3280A_Down & 0.0038 & 0.0038 & 0.0039 & 0.0045 & 0.0045 & 0.005 & 0.00097 & \\
\hline MPDL_3280A_Fluctuste & 0.0083 & 0.0084 & $8.1 e+05$ & 0.0076 & 0.0076 & 0.0076 & 0.0028 & \\
\hline MPDL3280A_Evolution & 0.011 & 0.0091 & 0.011 & 0.0091 & 0.0091 & 0.009 & 0.0043 & \\
\hline Cohort 1a (Squamous)_Down & 0.0007 & 0.00081 & 0.00069 & 0.00065 & 0.00065 & 0.00063 & 0.00015 & \\
\hline Cohort 1a (Squamous)_Fluctuate & 0.0022 & 0.0027 & 0.0009 & 0.0019 & 0.0019 & 0.0015 & 0.0041 & \\
\hline Cohort 1a (Squamous)_Evolution & 0.0013 & 0.0014 & 0.0013 & 0.0013 & 0.0013 & 0.0013 & 0.0017 & -0.006 \\
\hline Cohort to (Non-Squamous)_Up & 0.0063 & 0.0068 & 0.25 & 0.0068 & 0.0068 & 0.0068 & 0.00085 & \\
\hline Cohort 1b (Non-Squamous)_Down & 0.00046 & 0.00029 & 0.00047 & 0.00029 & 0.00032 & 0.00029 & 0.00011 & \\
\hline Cohort 16 (Non-Squamous)_Fluctuate & 0.0011 & 0.001 & 0.0021 & 0.001 & 0.0011 & 0.0011 & 0.00039 & \\
\hline Cohort ib (Non-Squamous)_Evolution & 0.0011 & 0.00059 & 0.0011 & 0.0011 & 0.0011 & 0.0012 & 0.0011 & \\
\hline Cohort 2 s (Squamous)_up & 0.0088 & 0.014 & 0.011 & 0.014 & 0.014 & 0.014 & 0.0059 & \\
\hline Cohort 2a (Squamous)_Down & 0.0012 & 0.00041 & 0.0013 & 0.00034 & 0.00032 & 0.00047 & 0.00044 & \\
\hline Cohort $2 a$ (Squamous)_Fluctuate & 0.011 & 0.011 & 0.015 & 0.011 & 0.011 & 0.011 & 0.011 & \\
\hline Cohort 2a (Squamous)_Evolution & 0.0078 & 0.007 & 0.0085 & 0.0078 & 0.0078 & 0.0082 & 0.0077 & \\
\hline Cohort 2b (Non-Squamous)_Up & 0.0052 & 0.0049 & $3.5 e+04$ & 0.0049 & 0.0049 & 0.0049 & 0.0028 & \\
\hline Cohort 2b (Non-Squamous)_Down & 0.0026 & 0.0021 & 0.0019 & 0.0022 & 0.0022 & 0.0025 & 0.00053 & 0.004 \\
\hline Cohort 2b (Non-Squamous)_Fluctuate & 0.0021 & 0.0019 & 0.0028 & 0.0019 & 0.0019 & 0.0019 & 0.00072 & \\
\hline Cohort 2b (Non-Squamous)_Evolution & 0.012 & 0.01 & 0.012 & 0.01 & 0.01 & 0.01 & 0.0077 & \\
\hline Cohort 3a (Squamous)_Up & 0.002 & 0.004 & $4.7 e+04$ & 0.0038 & 0.0038 & 0.0038 & 0.0012 & \\
\hline Cohort 3s (Squamous)_Down & 0.00037 & 0.00028 & 0.00038 & 0.00035 & 0.00039 & 0.00032 & 0.00015 & \\
\hline Cohort 3a (Squamous)_Fluctuate & 0.0067 & 0.0059 & $6.8 e+05$ & 0.0059 & 0.0059 & 0.0059 & 0.013 & \\
\hline Cohort 3a (Squamous)_Evolution & 0.0064 & 0.0056 & 0.0099 & 0.0064 & 0.0064 & 0.0068 & 0.006 & \\
\hline Cohort 3b (Non-Squamous)_Up & 0.011 & 0.0077 & $1.4 e+05$ & 0.0083 & 0.0082 & 0.0082 & 0.00089 & \\
\hline Cohort 3b (Non-Squamous)_Down & 0.0014 & 0.0018 & 0.0022 & 0.0016 & 0.0016 & 0.0023 & $6.9 \mathrm{e}-05$ & \\
\hline Cohort 3b (Non-Squamous)_Fluctuate & 0.0037 & 0.0028 & 0.0044 & 0.0029 & 0.0029 & 0.003 & 0.00063 & -0.002 \\
\hline Cohort 3b (Non-Squamous)_Evolution & 0.0026 & 0.0011 & 0.0026 & 0.00099 & 0.00099 & 0.00099 & 0.0037 & \\
\hline Docaiaxel_Up & 0.0045 & 0.0026 & 0.0064 & 0.0024 & 0.0024 & 0.0024 & 0.0013 & \\
\hline Docetaxel_Down & 0.0051 & 0.002 & 0.0054 & 0.0021 & 0.0021 & 0.0021 & 0.00085 & \\
\hline Docetaxel_Fluctuate & 0.012 & 0.0094 & $3.9 e+04$ & 0.0094 & 0.0094 & 0.0094 & 0.0014 & \\
\hline Docetaxel_Evolution & 0.0075 & 0.0057 & 0.0077 & 0.0059 & 0.0059 & 0.0059 & 0.0027 & \\
\hline MPDL3280A_Up & 0.029 & 0.015 & $1.6 e+04$ & 0.019 & 0.019 & 0.021 & 0.0017 & \\
\hline MPDL3280A_Down & 0.012 & 0.009 & 0.012 & 0.0091 & 0.009 & 0.0098 & 0.00033 & \\
\hline MPDL3280A_Fluctuate & 0.043 & 0.04 & $1.6 e+04$ & 0.041 & 0.041 & 0.041 & 0.0027 & \\
\hline MPDL_3280A_Evolution & 0.011 & 0.01 & 0.011 & 0.01 & 0.01 & 0.01 & 0.0027 & 00 \\
\hline
\end{tabular}

Figure 6: Mean absolute error values for prediction of tumor dynamics, fitted separately per model and category. A lower value corresponds to a better fit. 

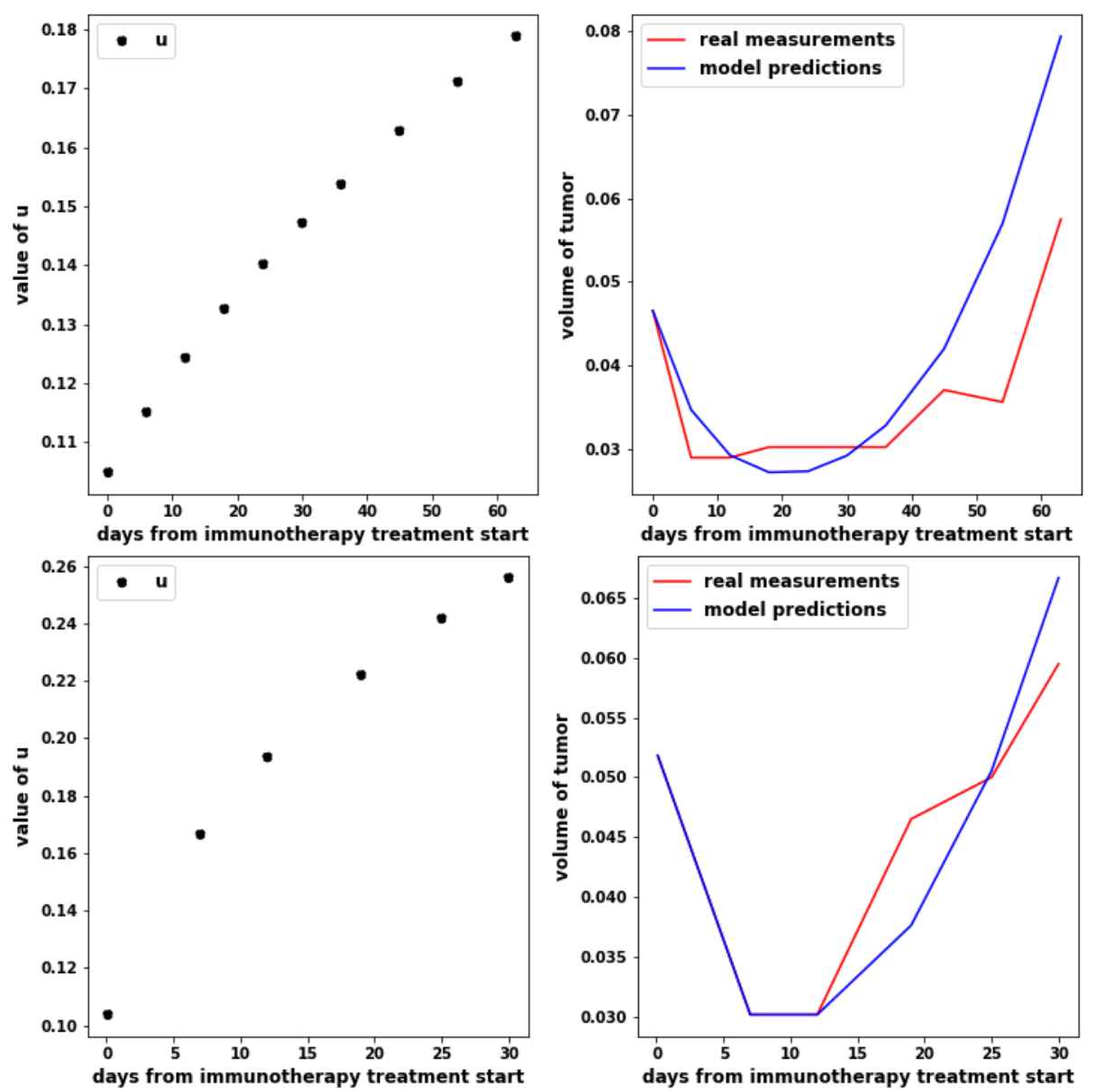

Figure 7: Predictions given for two tumors in the "evolution" category for a patient treated with immunotherapy (up) and a patient treated with Docetaxel (down). 

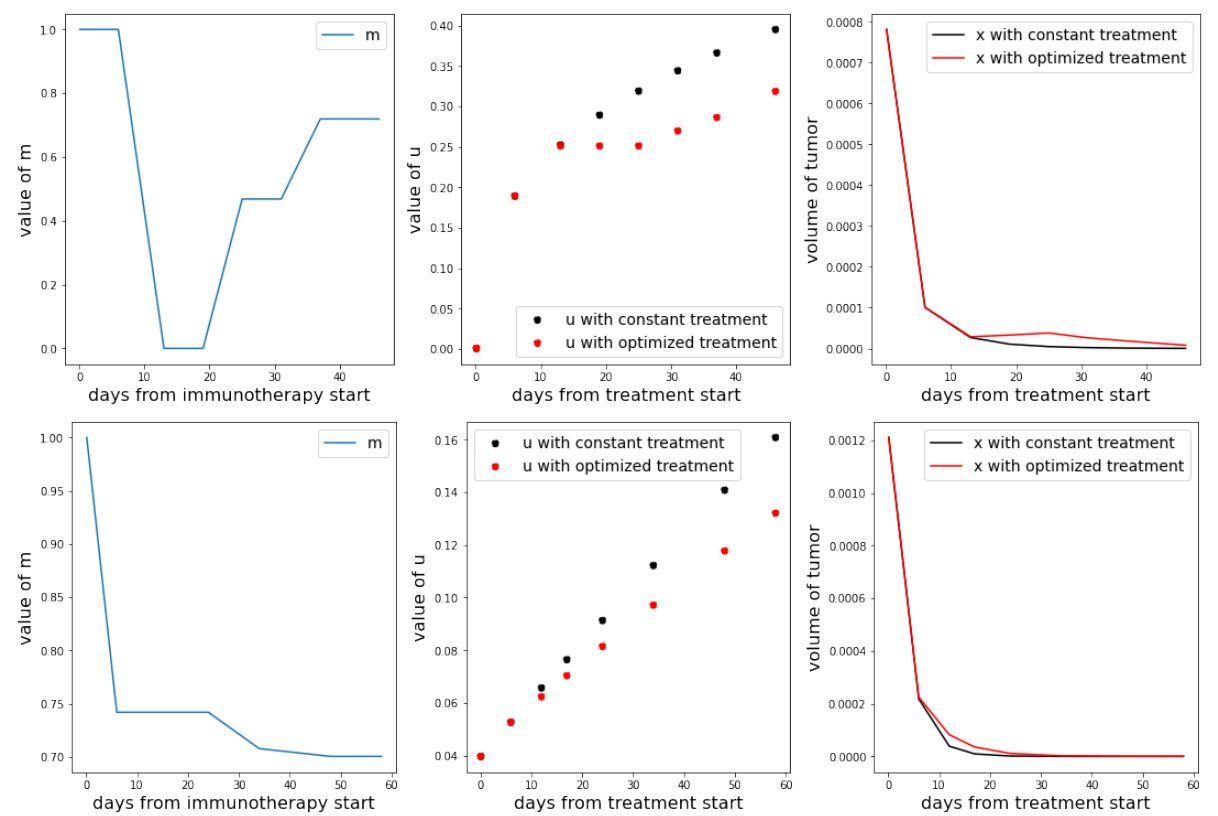

Figure 8: Comparison between continuous treatment and optimized treatment (with the aim of minimizing the final tumor burden) in a patient treated with chemotherapy.
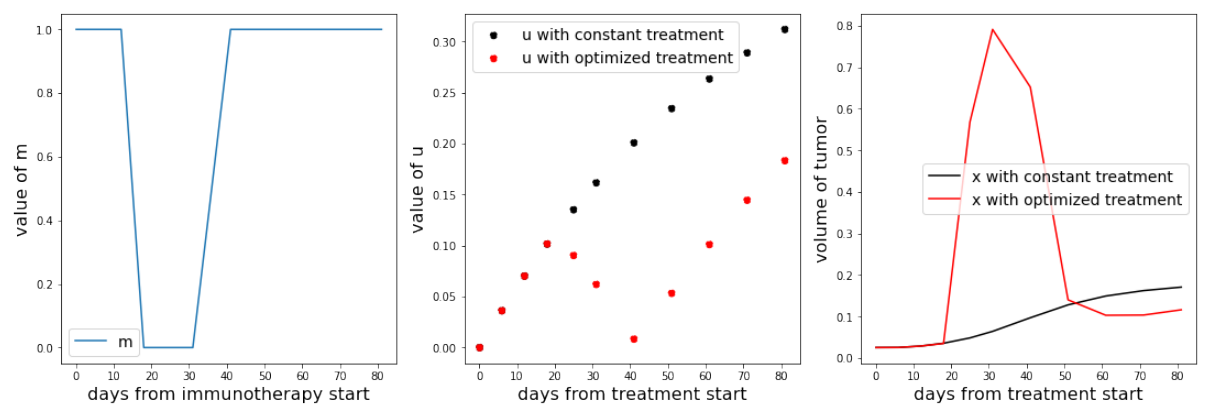

Figure 9: Comparison between continuous treatment and optimized treatment in a longer time period (with the aim of minimizing the final tumor burden) in a patient treated with immunotherapy.
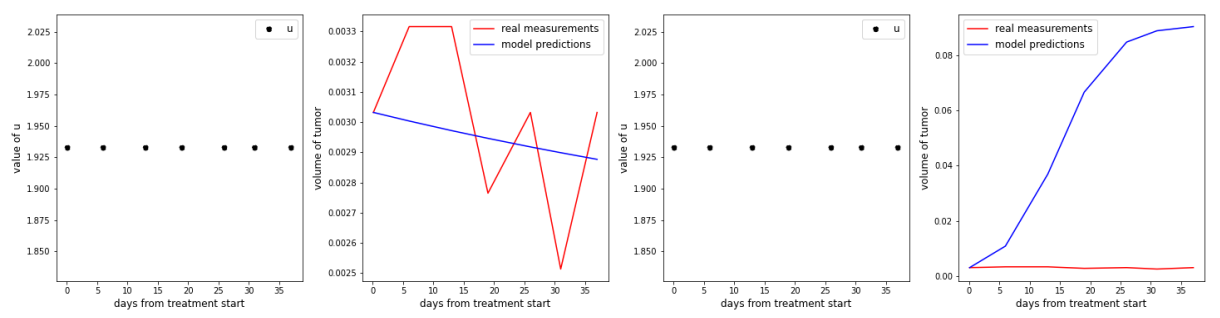

Figure 10: Real tumor evolution subject to Docetaxel (left) versus simulation of immunotherapy (right). 

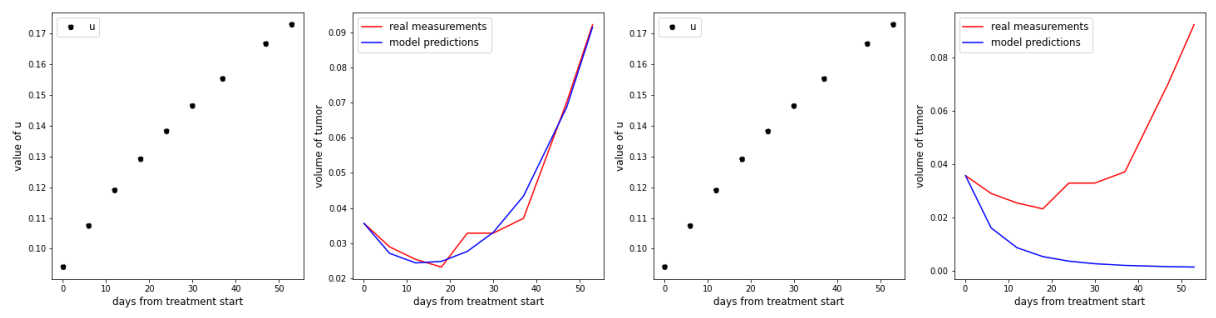

Figure 11: Real tumor evolution subject to immunotherapy (left) versus simulation of Docetaxel (right).
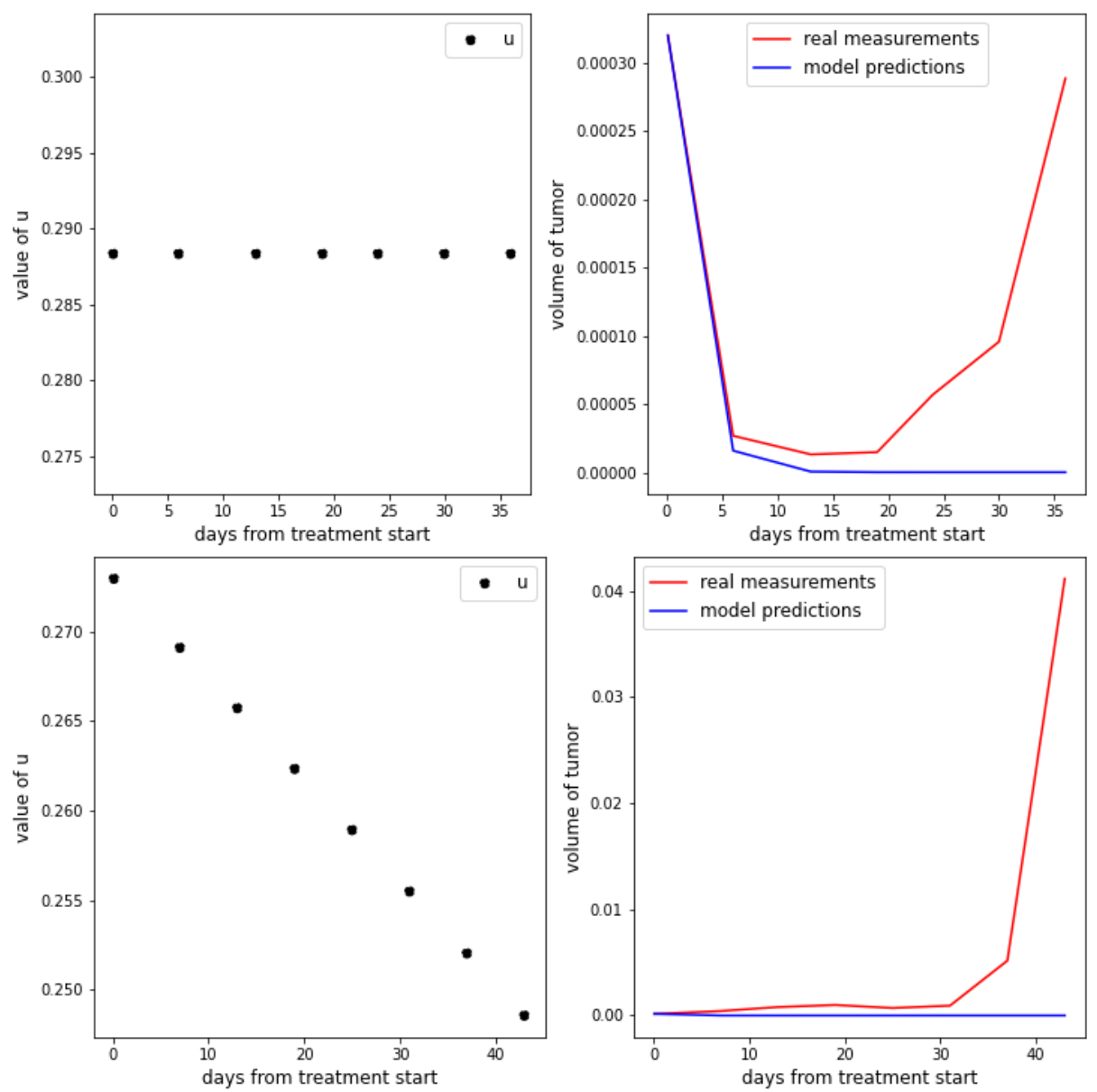

Figure 12: Evolution of tumor volume of two tumors in the category "Evolution" under an alternative treatment that targets evolution of resistance. 